\title{
Direct evidence for aerial egg deposition in the burrows of the Malaysian mudskipper, Periophthalmodon schlosseri
}

Atsushi Ishimatsu · Tatsusuke Takeda · Yuko Tsuhako · Tomas T. Gonzales · Khay Huat Khoo

A. Ishimatsu $(\quad) \cdot$ T. T. Gozales

Institute for East China Sea Research, Nagasaki University, Nagasaki 851-2213, Japan

T. Takeda

Department of Animal and Marine Bioresource Science, Faculty of Agriculture, Kyushu University, Fukuoka 812-8581, Japan

Y. Tsuhako

Department of Chemistry, Biology and Marine Science, Graduate School of Engineering, University of the Ryukyus, Okinawa 903-0213, Japan

K. H. Khoo

School of Biological Sciences, Universiti Sains Malaysia, 1180 Penang, Malaysia

Present Address:

T. T. Gonzales

Center for General Education, AMA International University - Bahrain, P.O. Box 18041, Manama, Kingdom of Bahrain 
Running head: Aerial egg deposition in mudskippers 


\begin{abstract}
The presence of mudskipper eggs in an air-filled chamber was confirmed by direct endoscopic observation of intact burrows of Periophthalmodon schlosseri in a mudflat in Penang, Malaysia. For all five burrows from which video images of egg chambers were successfully obtained, the presence of air was unequivocally demonstrated by the existence of an air-water interface inside the chambers. Of these burrows, eggs were found in two, but not in the others. Eggs were laid uniformly in a monolayer on the inner top surface of the chamber. The much brighter color of the surface mud of the egg chambers than the surrounding mud, irrespective of the presence or absence of the eggs, suggested that the surface mud had been oxidized by deposited air.
\end{abstract}

Key words: Aerial egg deposition, Burrows, Mudskippers, Periophthalmodon schlosseri

\title{
Introduction
}

It has been long been known that mudskippers (Periophthalmus argentilineatus, Periophthalmus crysospilos, Periophthalmus kalolo, Periophthalmus modestus, Boleophthalmus boddarti and Boleophthalmus. pectinirostris) lay their eggs in burrows (Clayton 1993). Mudskipper burrows often have some kind of diverticulum where eggs are usually found (Kobayashi et al. 1971; Brillet 1976; Ishimatsu et al. 1998a, 2000, 2007). Eggs are adhered to the walls and roofs of egg chambers with filamentous attachment threads, except in the case of P. argentilineatus (see Brillet 1976). However, the development of mudskipper embryos in the burrows has long been an enigma (Gordon 1995), since mudskipper burrows are always filled with severely hypoxic 
water that would not permit embryonic development (Ishimatsu et al. 1998a, b, 2000, 2007).

Our finding of air storage in mudskipper burrows sheds a new light on this question (Ishimatsu et al. 1998a). We have shown that variable volumes of gas are present in burrows of the Malaysian mudskipper, Periophthalmodon schlosseri (the volume ranging from 55 to over $4,000 \mathrm{ml}$ per burrow). The oxygen pressure $\left(\mathrm{PO}_{2}\right)$ of the gas varies widely but can be as high as the level in open air. More recently, our data on the Japanese mudskipper, $P$. modestus, indicated that egg-guarding males gulp mouthfuls of air and add them to the egg chamber during low tide when the mudflat is exposed. As a result, the $\mathrm{PO}_{2}$ of the egg-chamber gas increases continually during low tide, but declines during high tide, mainly due to embryonic oxygen consumption when the fish ceased transporting air (Ishimatsu et al. 2007). The presence of air in $P$. modestus egg chambers was also supported by the J-shaped burrow morphology, the distinct difference in mud color between the egg-chamber wall and other parts of the burrows, and the significantly higher redox potential of the egg-chamber mud compared with mud from other parts of the burrows.

These lines of evidence suggest that air storage in egg chambers is common among mudskippers as an adaptation that enables reproduction in the hypoxic mudflat substratum. Nonetheless, no direct evidence has been available for the presence of air and aerial embryonic development in an undisturbed egg chamber in mudskipper burrows. The application of analytical instrumentation (an $\mathrm{O}_{2}$ electrode, an endoscope, gas tubes and impedance electrodes) to this problem requires excavation down to the top of an egg chamber, exposing the egg-chamber lumen to ambient air (Ishimatsu et al. 2007). Therefore, one cannot rule out the possibility of artificial air trapping in egg 
chambers due to the procedure. The purpose of the present study was to obtain indisputable evidence for the presence of air in egg chambers and mudskipper embryos therein egg chambers by observing the interiors of intact mudskipper burrows with an endoscope. We have chosen P. schlosseri for this purpose because it excavates burrows that are large enough to manually insert an endoscope into the egg chamber (Ishimatsu et al. 1998a, 2000).

\section{Materials and methods}

Endoscopic burrow observations were made in a mudflat below the peninsular end of the Penang Bridge ( $5^{\circ} 21^{\prime} 18$ ’ N, $\left.100^{\circ} 2^{\prime} 36^{\prime \prime} \mathrm{E}\right)$ on the west coast of the Malay Peninsula. Burrows located within ca. $1 \mathrm{~m}$ from the shore were chosen for the observation. An industrial endoscope camera (Olympus, cable length $5 \mathrm{~m}$, outer diameter $7 \mathrm{~mm}$ ) was threaded through a vinyl pipe and directly inserted into the main vertical shaft of Periophthalmodon schlosseri burrows. The large diameter of the main vertical shaft (ca. 8-10 cm) allowed the insertion of an arm to position the tip of the endoscope CCD camera into a dome-shaped chamber located in the bottom horizontal tunnel (Fig. 1; see also Ishimatsu et al. 1998a). The bottom horizontal tunnels of the burrows we studied here were at 60-100 cm depth from the mudflat surface. The tip of the endoscope was initially covered with a plastic sheet, which was removed when the tip reached the egg chamber. Video images were recorded on a Hitachi VM-H200L 8 mm video recorder placed on the nearby beach. The images were later digitized using a Sony WV-D9000 video cassette recorder. Burrows of $P$. schlosseri can be easily distinguished from those of other animals by their large surface water pools (Ishimatsu et al. 1998a, b). Eggs collected from burrows were immediately tested for their hatchability by water 
immersion (Tsuhako et al. 2003).

\section{Results}

The main vertical shafts of Periophthalmodon schlosseri burrows were invariably filled with water to the level of the mudflat surface. Among eleven attempts, we succeeded in video recording five burrows. For the six unsuccessful attempts, we could not locate the dome-shaped chamber since the main vertical shafts of these burrows were too deep to reach the bottom horizontal tunnel. For all five successful burrows, the presence of air was unequivocally demonstrated by the existence of an air-water interface inside the dome-shaped chamber (Fig. 2a). Eggs were found in only two burrows, where they were laid uniformly in a monolayer on the inner top surface of the chamber (Fig. 2b). The mud surface of the egg chamber was bright in color, which is distinctly different from the much darker mud that appeared in the portion where surface mud was scraped off by accidentally touching it touch with a finger or an endoscope. A solitary burrow-guarding male (body weight $125.8 \mathrm{~g}$, total length $247.1 \mathrm{~mm}$ ) was recovered from one burrow. Embryos hatched immediately when egg collected from two burrows were immersed in seawater.

\section{Discussion}

The present results provide solid evidence for the presence of air and embryos in egg chambers of Periophthalmodon schlosseri. There has been always some degrees of uncertainty as to the aerial embryonic incubation of mudskippers, but the present observations unequivocally established the fact that embryos are incubated in an air-filled egg chamber in P. schlosseri, and presumably also in other mudskippers. 
There have been several fragmentary reports suggesting that mudskipper embryos develop in air-filled chambers. Kobayashi et al. (1971) stated that the egg chambers of Periophthalmodon. cantonensis (= P. modestus, see Murdy 1989) did not contain water upon excavation, and in most cases embryos were exposed to air inside the chambers, although they did not present evidence for the statement. Brillet (1976) described that burrows, except at the very bottom, of Periophthalmodon sobrinus $(=P$. argentilineatus) were filled with air during low tide so that embryos in the egg chamber were exposed to air. This contradicts our finding that the main shaft was always filled with water (Ishimatsu et al. 1998a, 2000, 2007). He also stated that the upward direction of the egg chamber was likely to favor air storage during burrow immersion, and conjectured that the stored air was used by burrow-guarding fish during high tide. In addition, Brillet (1976) reported that $P$. argentilineatus embryos, which had been incubated in the laboratory, hatched only if placed in air on moist mud, and immersed at more or less uniform intervals or when the embryos finished development. In spite of these early speculations, the aerial incubation of mudskipper embryos in an air-filled chamber has never been substantiated.

The present finding of aerial embryonic incubation in $P$. schlosseri raises new questions related to mudskipper reproductive strategy: (1) are eggs spawned prior to or after of the egg chamber is filled with air? (2) How do hatched larvae disperse out of the burrows? If spawning takes place before the egg chamber is filled with air, male and female fish must endure hypoxic conditions during reproduction, and air must be deposited rapidly thereafter. If spawning occurs after air filling, they must attach themselves in a supine position in the air to the inner top surface of the egg chamber. Recently, Shimizu et al. (2006) found that the intertidal rockhopper blenny Andamia 
tetradactyla spawns and inseminates the eggs in air, while they are attached to the undersides of air-exposed rock crevices. The finding of three egg chambers that contained air but no eggs suggests that the chambers are filled with air before spawning, as has been pointed for P. modestus (Ishimatsu et al. 2007), although it is hard to conceive of how adults of $P$. schlosseri (body weight up to 250g) can attach themselves in a supine position to the soft, wet mud surfaces of the ceilings of air-filled egg chambers during reproduction. Eggs of $P$. schlosseri are demersal, and have a clump of adhesive filaments at one end of the egg membrane (Tsuhako et al. 2003).

Hatching of $P$. modestus eggs is induced by immersion in water. At the end of an egg care period, a male repeatedly gulps the egg-chamber air and expels it through the main shaft of the burrow during high tide, which leads to the flooding of egg chambers and rapid hatching of the eggs (Ishimatsu et al. 2007). Since embryos hatched immediately when the collected P. schlosseri eggs were immersed, P. schlosseri presumably employs the same mechanisms of egg hatching. Newly hatched larvae of $P$. modestus were shown to succumb within $1 \mathrm{~h}$ if reared in hypoxic water simulating burrow $\mathrm{PO}_{2}$ conditions (Etou et al. 2007). Assuming a similar hypoxia tolerance for its larvae, rapid dispersion from inside the burrows is probably more critical for $P$. schlosseri. This is because the egg-chamber depth in P. schlosseri burrows (up to 120 cm, Ishimatsu et al. 2000) is far greater than those of other mudskippers (ca. 20-30 cm in $P$. modestus, Ishimatsu et al. 2007), despite the fact that the size of the hatched larvae is similar between mudskippers (P. modestus $2.8 \mathrm{~mm}$ total length, Kobayashi et al. 1972; B. pectinirostris 2.4-2.6 mm, Zhang et al. 1989; P. schlosseri 2.0-2.5 mm, Tsuhako et al. 2003). The side shaft, characteristic of $P$. schlosseri burrows, may play a role in the quick dispersion of the hatched larvae, although the opening of the side shaft 
is often clogged by mud deposition (Ishimatsu et al. 1998b). Periophthalmodon schlosseri is unusual among mudskippers in that two individuals are often observed in a burrow (Ishimatsu et al. 1998a, unpublished observation). This might indicate biparental care of the embryos, which may aid in the efficient addition of air during low tide and the rapid removal of hatched larvae from hypoxic burrows at the end of an egg incubation period. Further field studies are needed to answer these newly raised questions.

\section{Acknowledgements}

We would like to thank Mr. Takeshi Ichinoki and Ms. Naoko Itoki, Faculty of Fisheries, Nagasaki University, for their assistance in the field investigation. Our thanks are also due to Mr. Mutoo and Mr. Ng Chek Wan, School of Biological Sciences, Universiti Sains Malaysia for their help during the study. This study was financially supported by Monbusho International Scientific Research Program (08045057). This study complies with the current laws of Malaysia.

\section{References}

Brillet C (1976) Structure du terrier, reproduction et comportement des jeunes chez le poisson amphibie Periophthalmus sobrinus Eggert. Rev Ecol Terre Vie $30: 465-483$

Clayton DA (1993) Mudskippers. Oceanogr Mar Biol Annu Rev 31:507-577

Etou A, Takeda T, Yoshida Y, Ishimatsu A (2007) Oxygen consumption during embryonic development of the mudskipper (Periophthalmus modestus): 
implication for the aerial development in burrows. In: Fernandes MN, Rantin FT, Glass ML, Kapoor BG (eds) Fish respiration and environment. Science Publishers, Enfield, pp 83-91

Gordon MS (1995) Functional evidence from living vertebrates. In: Gordon MS, Olson EC (eds) Invasions of the land. Columbia University Press, New York, pp 216-250

Ishimatsu A, Hishida Y, Takita T, Kanda T, Oikawa S, Takeda T, Khoo KH (1998a) Mudskippers store air in their burrows. Nature 391:237-238

Ishimatsu A, Khoo KH, Takita T (1998b) Deposition of air in burrows of tropical mudskippers as an adaptation to the hypoxic mudflat environment. Sci Prog $81: 289-297$

Ishimatsu A, Aguilar NM, Ogawa K, Hishida Y, Takeda T, Oikawa S, Kanda T, Khoo KH (1999) Arterial blood gas levels and cardiovascular function during varying environmental conditions in a mudskipper, Periophthalmodon schlosseri. J Exp Biol 202:1753-1762

Ishimatsu A, Takeda T, Kanda T, Oikawa S, Khoo KH (2000) Burrow environment of mudskippers in Malaysia. J Biosci 11:17-28

Ishimatsu A, Yoshida Y, Itoki N, Takeda T, Lee HJ, Graham JB (2007) Mudskippers brood their eggs in air but submerge them for hatching. J Exp Biol 210:3946-3954

Kobayashi T, Dotsu Y, Takita T (1971) Nest and nesting behavior of the mud skipper, Periophthalmus cantonensis in Ariake Sound. Bull Fac Fish Nagasaki Univ $32: 27-40$

Kobayashi T, Dotsu Y, Miura N (1972) Egg development and rearing experiments of the larvae of the mud skipper, Periophthalmus cantonensis. Bull Fac Fish Nagasaki Univ 33:49-62 
Murdy EO (1989) A taxonomic revision and cladistic analysis of the oxudercine gobies (Gobiidae: Oxudercinae). Rec Austr Mus (Suppl) 11:1-93

Shimizu N, Sakai Y, Hashimoto H, Gushima K (2006) Terrestrial reproduction by the air-breathing fish Andamia tetradactyla (Pisces; Blenniidae) on supralittoral reefs. J Zool Lond 269:357-364

Tsuhako Y, Ishimatsu A, Takeda T, Khoo KH, Tachihara K (2003) The eggs and larvae of the giant mudskipper, Periophthalmodon schlosseri, collected from a mudflat in Penang, Malaysia. Ichthyol Res 50:178-181

Zhang Q-Y, Hong W-S, Dai Q-N, Zhang J, Cai Y-Y, Huang J-L (1989) Studies on induced ovulation, embryonic development and larval rearing of the mudskipper (Boleophthalmus pectinirostris). Aquaculture 83:375-385 


\section{Figure legends}

Fig. 1 Diagram of depicting the position of the endoscope inside a burrow of Periophthalmodon schlosseri. The endoscope was inserted by hand into the bottom of the main vertical shaft and, after reaching the air-water interface, the the CCD camera at the tip of the endoscope was raised to view the air-filled egg chamber. Note the surface opening of the side shaft is often occluded by mud deposition. awi: air-water interface, bht: bottom horizontal tunnel, $e$ endoscope, ec egg chamber, $m f s$ mudflat surface, mvs: main vertical shaft, ss side shaft, $s p$ surface pool, $s w$ side wall of the egg chamber. Scale $30 \mathrm{~cm}$.

Fig. 2 View of the air-water interface (awi; a) and the inner top surface (b) of a Periophthalmodon schlosseri burrow. A monolayer of spawned eggs was confirmed only on the inner top surface (b), not on the side wall (sw, a). The image in was taken using the endoscope CCD camera, which was raised above the air-water interface and bent obliquely downward toward the interface. Arrows in indicate the edge of the air-water interface. Scales in and represent 3 and $2 \mathrm{~cm}$, respectively. 


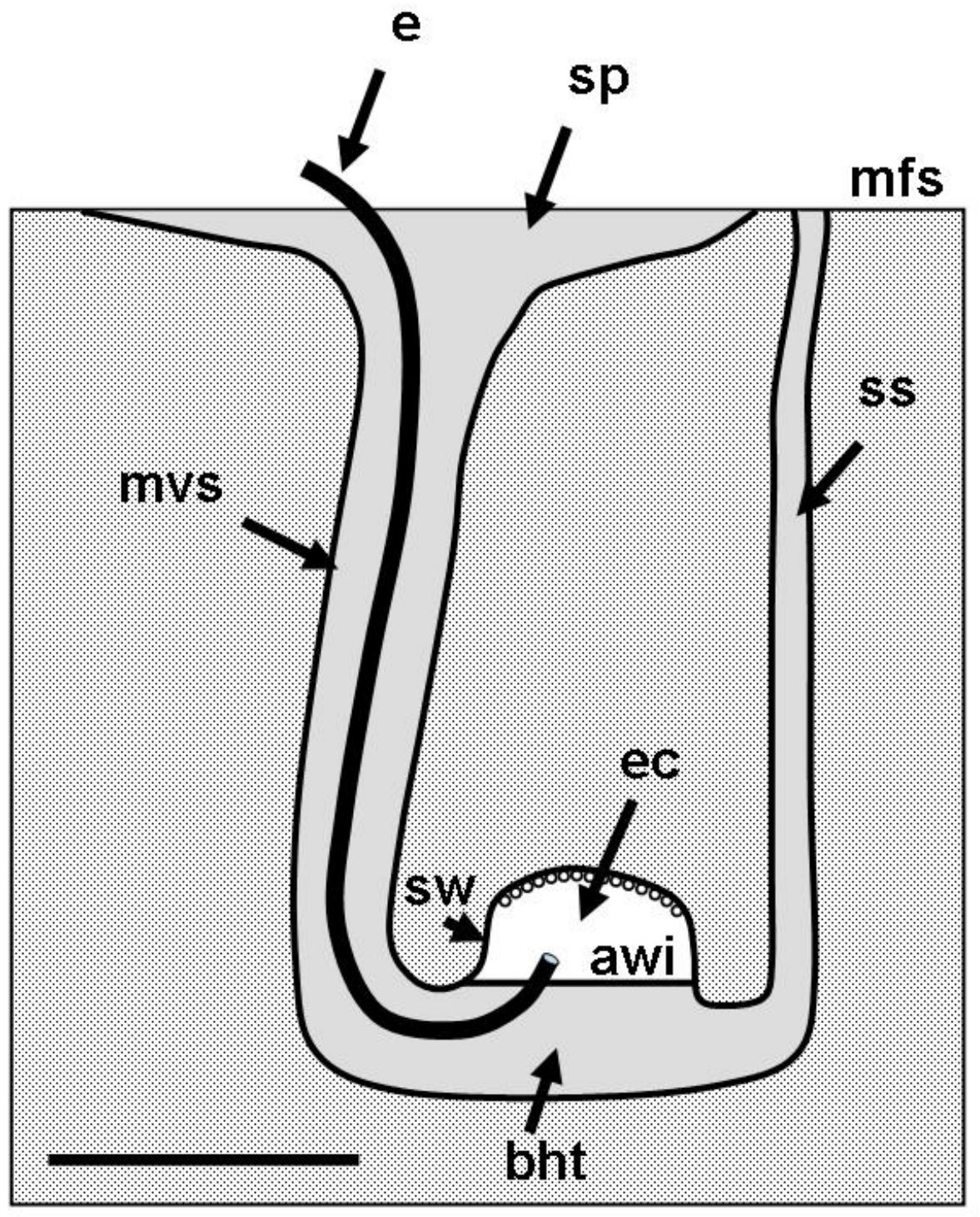

Fig. 1 


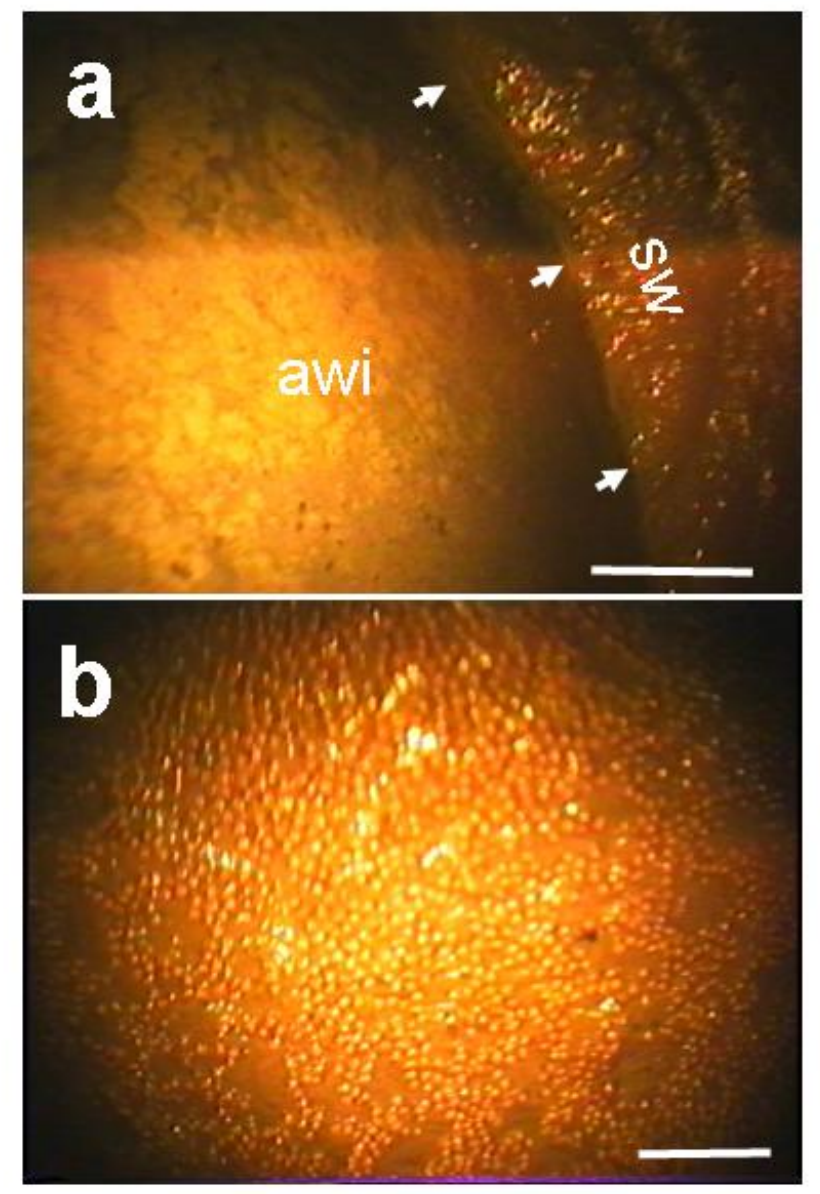

Fig. 2 